\title{
Engagement with ophthalmic simulation training has increased following COVID-19 disruption-the educational culture change required?
}

\author{
Sarah Campbell ${ }^{1}$ Jennifer Hind (1) ${ }^{1} \cdot$ David Lockington (1) ${ }^{1}$
}

Received: 30 January 2021 / Revised: 3 February 2021 / Accepted: 23 February 2021 / Published online: 11 March 2021

(c) The Author(s), under exclusive licence to The Royal College of Ophthalmologists 2021

COVID-19 has significantly disrupted normal medical education, including established ophthalmology training programmes. Trainees (and trainers) have experienced suspension of elective ophthalmic services, and even been redeployed to COVID-19 medical wards in some cases. High anxiety levels have been reported regarding the impact on ophthalmic training and the lack of clinical and surgical exposure during the year 2020 and beyond [1]. Local face-to-face teaching, regional, national and international ophthalmological conferences have had to be postponed (and then cancelled) due to social distancing requirements, inadequate space availability and travel restrictions and lockdowns. This has resulted in an explosion in online educational webinar events and replacement virtual meetings [2].

Ophthalmologists of all levels of training and seniority had their surgical sessions curtailed during the initial lockdown in March 2020, resulting in the Royal College of Ophthalmologists advising how to prioritise surgical cases in the setting of reduced resources, and how to restart elective services safely [3]. Pre-COVID, it was mandatory for new ophthalmic trainees in the UK to have attended an introductory microsurgical skills course at the Royal College of Ophthalmologists, London, and to have completed Modules $\mathrm{A}$ and $\mathrm{B}$ on the EYESI cataract simulator prior to engaging in any intra-ocular surgery. To maintain surgical skills during COVID-19 lockdowns, all trainees and trainers were also encouraged to engage with and utilise the various simulation options available, particularly during clinical sessions which had been cancelled. Currently, there has not

David Lockington

davidlockington@hotmail.com

1 Tennent Institute of Ophthalmology, Gartnavel General Hospital, Glasgow, UK been a mandatory requirement for either trainees or trainers to complete Module $\mathrm{C}$ or the more complex/complicationbased Module D. As clinical activity began to resume over the summer period in 2020, it became apparent that seniors and juniors were making greater use of the EYESI simulator in Glasgow than previously. We wanted to objectively evaluate if this impression of increased simulation use was accurate, and if it was maintained.

In January 2021, we contacted VRmagic Holding AG (Mannheim, Germany; makers of the EYESI simulator) and obtained the statistics for EYESI usage in Glasgow, UK, over the past 2 years. We agreed to use data from only individually registered users (so ignoring occasions the simulator was accessed through the anonymous "demo user" setting) and actual training time (from insertion of instruments until task was stopped) as duration of simulation use. The VRmagic team advised 'time in front of the simulator' (including navigating the courseware, reading task descriptions and end of task evaluations) could add at least $40 \%$ to the duration of use results. As all those tasks are not actual surgical simulation, we used the minimum training time values for a more accurate evaluation of learning.

In 2019, the EYESI simulator was accessed on a mean number of 16 occasions per month (median 15, range 3-43). This correlated to a monthly median duration of actual simulation training of $719.5 \mathrm{~min}$ (mean 798.6, range 75-2063) in that year. In 2020, the EYESI simulator was accessed on a mean number of 29 occasions per month (median 31, range 0-60). This correlated to a monthly median duration of actual simulation training of $1246.5 \mathrm{~min}$ (mean 1540.75, range 0-3441) in that year. There was a statistically significant increase in the monthly mean number of minutes of EYESI usage comparing each month between 2019 and 2020 (one way ANOVA; $F=5.42, p=0.029$ ) (see Fig. 1). 
Fig. 1 Graph showing weekly EYESI simulator usage in minutes in Glasgow, UK across the years 2019 and 2020

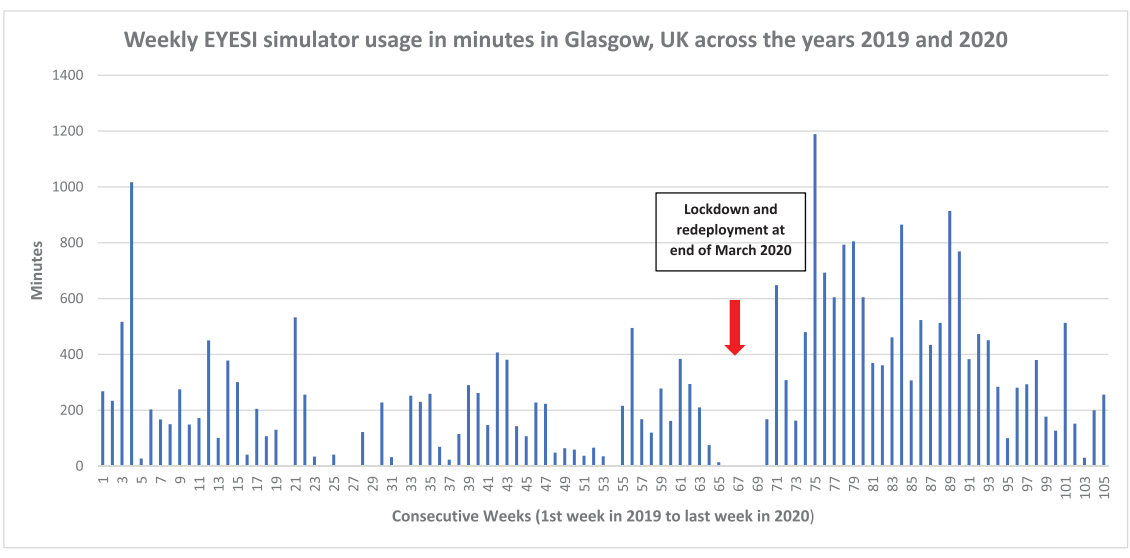

These statistics show that the level of engagement with simulation training increased in 2020 following COVID-19 redeployment of ophthalmic trainees in Glasgow (arrow in Fig. 1) compared to the previous year (2019) and has been maintained over the past 6 months at a significantly higher level than previously. We believe that the COVID-19 disruptions to normal job plans and educational activities have demonstrated the benefits of engaging with simulation training and skill maintenance to both the trainees and trainers. However, engagement with simulation should have already been a central pillar of best ophthalmic practice, irrespective of lockdowns and clinical service suspensions. Ensuring competence is maintained for surgical tasks should preserve confidence in one's ability to deal with complex and complicated surgical procedures. Regular simulation engagement offers that level of security $[4,5]$. It is our recommendation that as training programmes restart, the benefits and role of ophthalmic simulation will be enshrined, expanded and empowered to ensure safer surgical experiences for the trainees, trainers and the patients (most importantly). We call on all relevant stakeholders to provide the necessary time and dedicated finance required to provide and persist with this essential training facility, acknowledging that ophthalmic simulation training has been shown to be cost saving compared to the immediate and legacy costs of addressing avoidable surgical complications $[6,7]$.

\section{Compliance with ethical standards}

Conflict of interest Remaining authors declare no competing interests. DL is the ophthalmic Simulation Lead for the West of Scotland region at the time of submission.
Publisher's note Springer Nature remains neutral with regard to jurisdictional claims in published maps and institutional affiliations.

\section{References}

1. Ferrara M, Romano V, Steel DH, Gupta R, Iovino C, van Dijk EHC, et al. Reshaping ophthalmology training after COVID-19 pandemic. Eye. 2020;34:2089-97. https://doi.org/10.1038/s41433020-1061-3.

2. Chatziralli I, Ventura CV, Touhami S, Reynolds R, Nassisi M, Weinberg T, et al. Transforming ophthalmic education into virtual learning during COVID-19 pandemic: a global perspective. Eye. 2020:1-8. https://doi.org/10.1038/s41433-020-1080-0.

3. Royal College of Ophthalmologists. Restarting and redesigning of cataract pathways in response to the COVID 19 pandemic. London: Royal College of Ophthalmologists; 2020. https://www.rcophth.ac. uk/wp-content/uploads/2020/08/Resumption-of-Cataract-ServicesCOVID-August-2020-2.pdf. Accessed 27 Jan 2021.

4. Nairn J, Benjamin L, Lockington D. Comment on: 'Ophthalmology Specialist Trainee Survey in the United Kingdom'. The need to increase familiarity with the management of predictable cataract surgery complications via simulation-ensuring competence to improve confidence. Eye. 2020;34:1713-4. https://doi.org/10.1038/ s41433-019-0673-y.

5. Mulholland C, Lockington D. Trainee experience with capsular tension rings in Scotland-the need for structured simulation exposure to surgical adjuncts. Eye. 2020;34:1497-8. https://doi. org/10.1038/s41433-020-0856-6.

6. Ferris JD, Donachie PH, Johnston RL, Barnes B, Olaitan M, Sparrow JM. Royal College of Ophthalmologists' National Ophthalmology Database study of cataract surgery: report 6 . The impact of EyeSi virtual reality training on complications rates of cataract surgery performed by first and second year trainees. $\mathrm{Br} \mathrm{J}$ Ophthalmol. 2020;104:324-9. https://doi.org/10.1136/bjophthalmol-2018-313817.

7. Jamison A, Benjamin L, Lockington D. Quantifying the real-world cost saving from using surgical adjuncts to prevent complications during cataract surgery. Eye. 2018;32:1530-6. https://doi.org/10. 1038/s41433-018-0133-0. 